\title{
Research on the Catalyst Used for Vertical Aligned Carbon Nanotube Arrays Prepared by Catalytic Chemical Vapor Deposition
}

\author{
Zhao Jingze, Liu Rongjun*, Wang Yanfei, Cao Yingbin \\ College of Aerospace Science an Engineering, Science and Technology on Advanced Ceramic Fiber and Composites Laboratory, National \\ University of Defense Technology, Changsha, China
}

\author{
Email address: \\ rongjunliu@126.com (Liu Rongjun) \\ ${ }^{*}$ Corresponding author
}

\section{To cite this article:}

Zhao Jingze, Liu Rongjun, Wang Yanfei, Cao Yingbin. Research on the Catalyst Used for Vertical Aligned Carbon Nanotube Arrays Prepared by Catalytic Chemical Vapor Deposition. Science Discovery. Vol. 6, No. 4, 2018, pp. 231-242. doi: 10.11648/j.sd.20180604.12

Received: May 21, 2018; Accepted: July 12, 2018; Published: July 20, 2018

\begin{abstract}
Process control of the oriented growth of vertical aligned carbon nanotube arrays and preparation of various ordered structure of carbon nanotube films is the major challenge for practical application. In order to better understand the parameters governing the catalytic properties of the catalytic nanoparticles, this paper summarizes the advances in the catalyst used for vertical aligned carbon nanotube arrays prepared by catalytic chemical vapor deposition, and the process parameters were considered to be able to control the particles size, composition, structure of catalyst. And investigations into the effects of during time, temperature, atmosphere in pre-treatment process, and the transition layer on catalyst morphology evaluation were further found that the catalyst particles size, distribution and density can be transformed through the surface diffusion and inter-layer diffusion influenced by those process.
\end{abstract}

Keywords: Catalytic Chemical Vapor Deposition, Vertical Aligned Carbon Nanotube Arrays, Catalyst, Structure Tailoring

\section{CCVD法制备垂直碳纳米管阵列的催化剂研究}

赵静泽, 刘荣军 ${ }^{*}$, 王衍飞, 曹英斌

国防科技大学, 空天科学学院陶瓷纤维及其复合材料重点实验室, 长沙, 中国

\section{邮箱}

rongjunliu@126.com(刘荣军)

摘要：控制垂直碳纳米管阵列的取向生长，制备各种有序阵列结构的碳纳米管薄膜是实现碳纳米管实际应用的重要问 题。为了更加深入了解催化剂纳米颗粒的参数控制, 本文概述了近年来, 应用于催化化学气相沉积（CCVD）法制备 垂直碳纳米管阵列催化剂的制备研究进展, 指出制备参数能够控制催化剂的粒径分布, 成分和结构。探讨了退火处理 中时间、温度和气氛以及缓冲层对催化剂形貌演变的影响, 指出上述因素主要引起催化剂在表面层扩散以及向基底层 扩散的变化, 来改变催化剂的分布及尺寸。

关键词: 催化化学气相沉积, 垂直碳纳米管阵列, 催化剂, 结构调控 


\section{1. 引言}

碳纳米管中碳原子的 $\sigma-\pi$ 再杂化使管外电子云密度 大大增加, 从而赋予了碳纳米管优异的热、光、电和力学 等性质 [1]。垂直碳纳米管阵列(CNTAs), 是碳管垂直于基 底生长, 形成平行紧密排列的宏观结构。阵列中的碳纳米 管具有较一致的长径比, 良好的取向、较高的纯度等优点, 为碳纳米管以功能器件形式获得应用提供了可能, 广泛地 应用于纳米电子器件[2], 超级电容器电极[3]、黑体材料[4]、 吸波材料 [5]和热界面材料[6]。另外, 特殊的 CNTAs可以 通过干法抽丝、抽膜直接形成连续的碳纳米管纤维或者薄 膜 $[7,8]$ 。

不同应用领域对碳纳米管阵列中碳纳米管的直径, 管 壁数以及面密度用不同的要求。例如, 在黑体材料中, 需 要控制碳纳米的面密度以及空间填充率 (管直径与管间距 的比例), 以实现陷光和光吸收的目的。文献报道显示, 具有超低反射性质的垂直碳纳米管阵列每平方厘米碳纳 米管数的数量级通常达到 $10^{11}$ 个, 管直径 $20 \mathrm{~nm}$ 左右 [9-11] (多为单壁碳或者双壁碳纳米管);而应用于水体净化的碳 纳米管阵列的管间距甚至达到 $10 \mathrm{~nm}$ 以下 $[12]$ 。另外, 上述 应用对碳纳米管阵列的纯净度、结晶度及准直性还有一定 要求。最重要的是, 低成本批量化生产是影响其能否实现 广泛应用的重要因素。

在众多制备技术中, 催化化学气相沉积 (CCVD) 工 艺简单, 成本低且可控性好, 已成为实验室及工业上制备 CNTAs的最主要的方式。主要方法有浮动催化法和预制催 化法。浮动催化法是量产多壁碳纳米管阵列的主要方法, 设备简单, 过程中催化剂形成和碳纳米管生长协同进行, 但反应的复杂性增加了精准控制的难度。预制催化法按照 基板的不同, 分为两类。一，介孔基底 [13], 以其孔道结 构为模版并结合高效的催化剂, 从而获得特定规格的碳纳 米管阵列。但祄底材料需要特殊处理, 大大增加了工艺成 本及操作复杂度。二, 平板基底, 通常在单晶硅 $\mathrm{Si}$ 、二氧 化硅、石英、石墨纸、铜、铝等平坦基底[14-19]上沉积纳 米级的催化剂薄膜或者高密度催化剂纳米颗粒, 一定预处 理后获得高催化活性的纳米颗粒, 制备出准直性量好的垂 直碳纳米管阵列。研究证明催化剂的尺寸、分布、结构组 成等是影响碳纳米管阵列的重要因素, 因此控制催化剂的 制备及处理有助于实现CNTAs控制生长。

催化剂作为CCVD工艺中关键点, 在制备碳纳米管工艺 中有着众多且复杂的影响因素, 我们无法在本综述中详尽阐 述。国内外研究者关于碳纳米管阵列研究的综述文章众多, 例如, 最新制备方法, 理论模拟, 催化剂的角色, 手性控制 等[19-24]。本文着重介绍了近年来关于催化剂的制备工艺以 及后续处理对其形貌及性能等方面影响的研究, 从而产生对 碳纳米管阵列生长影响的研究成果, 达到对催化剂更进一步 的理解和认知, 为未来科研工作的开展提供便利。

\section{2. 催化剂的种类}

催化剂作为碳源分解活性中心及碳沉积成核中心, 碳 纳米管的长出涉及到碳在催化剂颗粒上的表面扩散及体
扩散 [25]。目前, 文献报道的具有催化活性的催化剂种类 主要有四种。（一）过渡金属单质及合金。近十几年报道 的Fe, Co, Ni, Pd, Pt, Au, Mn, W, Ti, Co, Mg, Al, In, Na, K, Cs等金属能够用于MWCNT的制备, 能催化生长出CNT的 纳米颗粒的直径通常大于 $3 \mathrm{~nm}$; 单壁碳纳米管的常用 $\mathrm{Fe}, \mathrm{Co}, \mathrm{Ni}, \mathrm{Pd}, \mathrm{Pt}, \mathrm{Ru}, \mathrm{Cu}, \mathrm{Ag}, \mathrm{Au}, \mathrm{Mn}, \mathrm{Cr}, \mathrm{Mo}$ 等金属做催化, 纳米颗粒的粒径往往小于 $3 \mathrm{~nm}$ 。早在 2004 年, Deng[26]等 人就通过量子理论模拟发现, 在碳形核阶段单金属催化活 性大小依次为 $\mathrm{Mo} 、 \mathrm{Cr} 、 \mathrm{Co} 、 \mathrm{Pt} 、 \mathrm{Fe} 、 \mathrm{Ni} 、 \mathrm{Cu}$; 在碳管的生 长阶段, 催化剂的活性大小依次为Fe、Ni、Mo、Co、Pt、 $\mathrm{Cr} 、 \mathrm{Cu}$ 。2014年, Yuki[27]等人发现在 $\mathrm{Al}_{2} \mathrm{O}_{3} / \mathrm{SiO}_{2} / \mathrm{Si}$ 基底 上 $\mathrm{Fe}$ 薄膜相较于 $\mathrm{Co}$ 薄膜能够在更薄和更厚的厚度上实现 对碳纳米管的催化生长, 作者通过研究覆盖在金属表面的 石墨层发现，铁颗粒表面能够覆盖更多有缺陷的石墨。一 些合金催化剂能够弥补单一金属的催化缺陷, 达到更好的 催化效果。Deng发现制备SWCNT的催化剂活性顺序为 Ni $+\mathrm{Co}>\mathrm{Ni}+\mathrm{Pt}>\mathrm{Ni}>\mathrm{Ni}+\mathrm{Cu}$, 得到了实验及理论模拟的 一致验证。某些惰性或者低活性金属能够通过合金化而拥 有较高的催化活性。2014年, Yang等人[28]用溶胶凝胶法 制备的 $\mathrm{Ni}-\mathrm{Cu} / \mathrm{MgO}$ 催化剂使得多壁碳纳米管阵列的产率 在 $650^{\circ} \mathrm{C}$ 下提升了 $927 \%$, 产率的提升就归功于 $\mathrm{Cu}$ 对活性 $\mathrm{Ni}$ 还原反应的有效提升。(二) 氧化物 $\mathrm{SiO}_{2} 、 \mathrm{Al}_{2} \mathrm{O}_{3} 、 \mathrm{TiO}_{2}$ 、 $\mathrm{ZrO}_{2} 、 \mathrm{Fe}_{\mathrm{x}} \mathrm{O}_{\mathrm{y}}$ 。（三）陶瓷纳米颗粒: $\mathrm{Fe} 3 \mathrm{C}, \mathrm{SiC}$ 等。(四) 纳米颗粒: $\mathrm{Si} 、 \mathrm{Ge} 、 \mathrm{C}$ 等。众多催化剂中, 过渡金属 $\mathrm{Fe}$ 、 Co、 $\mathrm{Ni}$ 由于具有相对较高的碳溶解度和碳扩散系数, 有利 于碳纳米管的成核与生长 [29], 成为了CVD工艺中研究最 多、应用最广的高效催化剂。

\section{3. 催化剂制备工艺}

催化剂的制备工艺本身会对催化剂的形貌和活性产 生重要影响。目前, 主要应用于制备碳纳米管阵列催化剂 的方法主要有物理法和化学法。

\subsection{PVD}

物理气相沉积（PVD）方法技术成熟, 稳定性高, 制 备的催化剂具有较高的催化活性, 已成为制备催化剂的主 流方式。PVD法制备的催化剂通常为薄膜状, 不同制备工 艺, 催化剂薄膜的控制, 表面的粗㮞性, 与基底附着力等 均有所差别。目前, 主要的制备工艺有: 溅射、原子层沉 积、真空蒸镀法、分子外延等。

\subsection{1. 溅射沉积}

溅射是在一定真空状态下, 利用高能粒子轰击靶材表 面，而使固体分子或者原子从表面射出，在基片上沉积形 成薄膜。控制溅射工艺过程的功率及时间就可以控制溅射 速率和薄膜材料的厚度。

Yamada等[30]通过溅射法精准控制催化剂 $\mathrm{Fe}$ 薄膜的 厚度, 在1.6 1.9nm厚范围内高效合成高纯度的双壁碳纳 米管阵列; 其中催化剂 $\mathrm{Fe}$ 在 $1.69 \mathrm{~nm}$ 时, 可获得 $85 \%$ 的双壁 碳纳米管, 平均管径在 $3.75 \mathrm{~nm}$ 。Lee等人[31]利用射频磁控 溅射法在 $\mathrm{Si}$ (001) 基底上成功制备了 $\mathrm{Fe}-\mathrm{Al} 2 \mathrm{O} 3$ （铁占 
5\% 20wt\%）用于碳纳米管生长，研究表明：催化剂制备 过程中, 射频功率功率减少会使催化剂粒径减小从而导致 碳纳米管直径减小。2016年，Jafarpour等人[32]用不同厚 度的Co-Mo双分子层制备SWCNT时发现，低的离子浅射 沉积速率制备的催化剂在经过氢气退火处理后具有更小 的粒径, 且粒径分布也更加集中。当复合催化剂薄膜厚度 增大后, 这种差异性更加明显, 如图1。作者认为主要是 由于低沉积速率降低了基底表面成核量, 表面小颗粒聚集 形成“山谷”状大颗粒的趋势减小。除了形貌的控制, 调整 溅射工艺可以改变催化剂的结构。Nesov[33]在溅射腔内沉

\section{(a) High deposition rate}

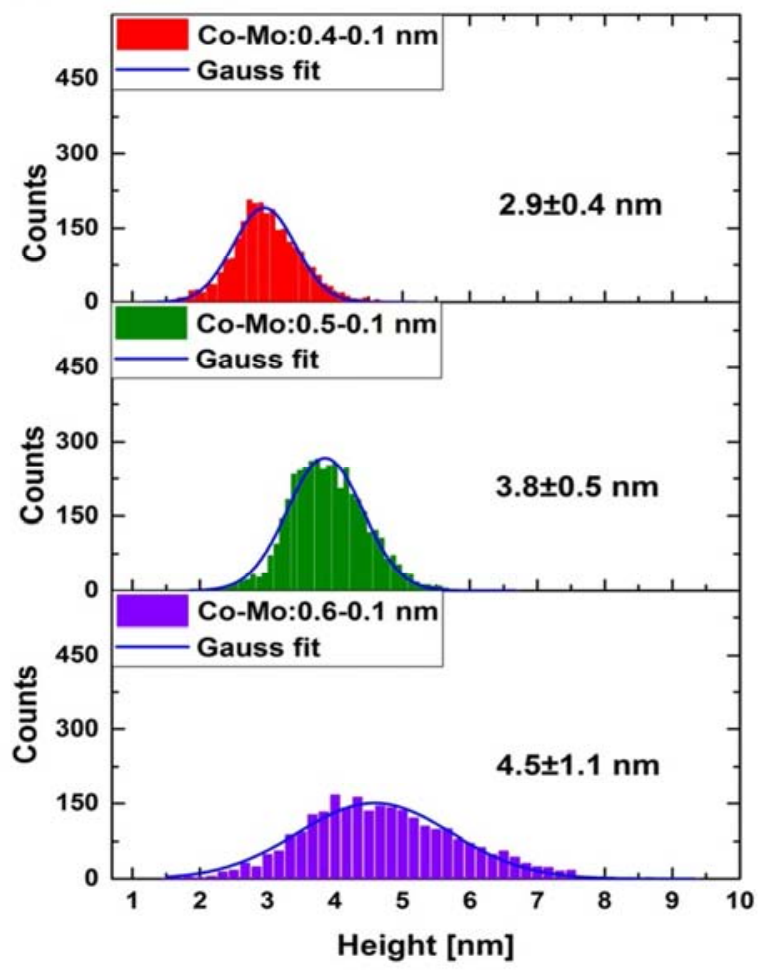

积Ni催化剂的同时引入甲烷, 通过甲烷气体含量的变化获 得不同含 $\mathrm{Ni}$ 量的 $\mathrm{nc}-\mathrm{Ni} / \mathrm{C}$ 薄膜, 研究发现催化剂化学组成会 直接影响催化剂纳米颗粒的微观形貌。Ohashi[34]利用 $\mathrm{MgO}$ 与 $\mathrm{FePt}$ 晶格的相近性, 在单晶 $\mathrm{MgO}$ 基底上交替溅射 $\mathrm{FePt}(0.25-1 \mathrm{~nm}) /[\mathrm{MgO}(0.5-1 \mathrm{~nm}) / \mathrm{FePt}(0.25-1 \mathrm{~nm})] \mathrm{X}(\mathrm{X}=1$ 或2)制备了 $\{111\}$ 取向生长的八面体结构FePt纳米颗粒, 催化生长出具有金属性的单壁碳纳米管阵列。值得注意的 是这种独特的制备工艺使得催化剂具有了独特的催化性 能。

\section{(c) Low deposition rate}

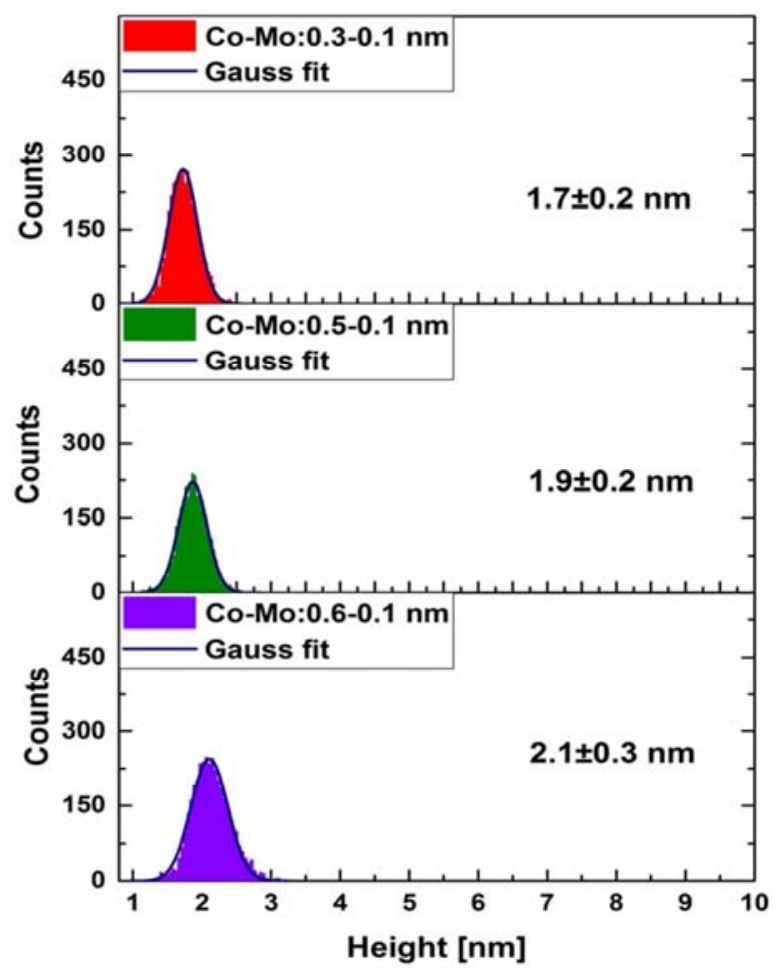

图 $1 \mathrm{Al}_{2} \mathrm{O}_{3}$ 支撑层上不同沉积速率获得不同厚度 $\mathrm{Co}-\mathrm{Mo}$ 催化剂薄膜经氢气退火处理后平均粒径及粒径分布 [32]。

\subsection{2. 原子层沉积}

ALD技术是一种在速率可控的条件下，通过前驱体和 反应气体选择性地脉冲进入腔体到达基底, 在其表面发生 物理和化学吸附和化学反应, 将物质以单原子膜的形式一 层一层沉积在基底表面的方法[35]。原子层沉积可在不规 则和多孔基底上大面积制备均匀密集且具有优异保形性 氧化物薄膜[36]。目前, 利用ALD工艺制备用于碳纳米管 生长的催化剂的种类相对较少。

2014年, Chen[37]在 $\mathrm{Si} / \mathrm{SiO}_{2}(200 \mathrm{~nm})$ 基底上用叔丁基二 茂铁（TBF）和 $\mathrm{O}_{3}$ 制备了表面粗楉度约为 $0.2 \mathrm{~nm}$ 的 $\mathrm{Fe}_{2} \mathrm{O}_{3}$ 薄 膜,in-situ和ex-situ XPS图谱表明TBF在ALD工艺中仅发生 了部分分解, 而这种不纯净的催化剂却能生长出高密度的 碳纳米管阵列。随后2015年, 杨[38]等人使用二甲基铝, 三氯化铁和水为先驱体, 在单晶硅(100)衬底上同时制备了 $\mathrm{Al}_{2} \mathrm{O}_{3}$ 缓冲层和纯净度高的 $\mathrm{Fe}_{2} \mathrm{O}_{3}$ 催化剂, 但有较大的表面 粗䊁度。这说明ALD工艺中催化剂前驱体对薄膜的形貌和
成分有影响。杨还深入探究发现薄膜的沉积厚度通过控制 反应的循环次数, 且催化剂厚度增加导致形成的纳米颗粒 的粒径增大，而CNTs的管壁数和管径都增大。Tang[39] 等人在蓝宝石上用 TMA 和水为先驱体制备的 $1 \mathrm{~nm}$ 厚的 $\mathrm{Al}_{2} \mathrm{O}_{3}$ 薄膜, 直接催化生长管径为 $1.26 \pm 0.033 \mathrm{~nm}$ 的SWCNT。

原子层沉积法易于制备厚度更小的催化剂薄膜, 更好 地应用于单壁碳纳米管阵列的制备。另外，前驱体沉积的 自终结反应发生在基底表面, 使薄膜材料在基底有较高的 附着性。

\subsection{3. 真空蒸镀法}

真空蒸镀加热的方式主要有, 电子束加热、射频加热、 电阻加热等。电子束蒸镀 (亦或电子束气相沉积) 是较为 常用的一种模式。电子束蒸发镀膜技术是在真空条件下利 用电子束直接加热蒸发材料, 使蒸发材料气化后向基板运 输并在基底上凝结形成薄膜的方法 [40]。蒸镀材料的厚度 
控制依赖操作人员的沉积经验, 在可控性上, 电子束蒸镀 技术显得较差。

Yoshikawa 等 [41] 利用电子束加热分别沉积了 $\mathrm{Fe}(0.5 \mathrm{~nm})$ 和 $\mathrm{Co}(1 \mathrm{~nm})$, 催化乙醇于 $660^{\circ} \mathrm{C}$ 和 $3.3 \mathrm{Kpa}$ 压力下 制备了由SWCNT、DWCNT和TWCNT组成, 易从柔性铝 䈃上剥落的碳纳米管阵列。Zhang[42]等人改进垂直碳纳米 管阵列的“催化剂沉积-碳纳米管生长-阵列增密”循环增密 工艺时发现, 每次循环中第一步电子束蒸镀铁/氧化铝过 渡层的沉积电压过大会导致碳纳米管阵列结构破坏, 而电 压决定着催化剂复合层的沉积速率, 从而影响到循环工艺 中碳纳米管阵列密度。

\section{2. 化学方法}

\subsection{1. 金属一有机化合物化学气相沉积(Metalorganic Chemical Vapor Deposition)}

金属有机化合物化学气相沉积（MOCVD）是将金属 有机化合物先驱体加热气化后通过载气载入高温反应腔 内, 高温条件下发生裂解并在基底表面沉积[43]。铁、镍、 钴为金属配位中心的金属有机化合物是MOCVD工艺最常 用的先驱体。

以铁为例, 常用的先驱体的种类有羰基铁、茂基铁、 其衍生物。根据 Thomas Duguet等人[44]的报道, 仅仅 $0.000317 \mathrm{mmo}$ 的 $\mathrm{Fe}_{3}(\mathrm{CO})_{12}$ 就可以在硅基底上沉积 $10 \mathrm{~nm}$ 的 $\mathrm{Fe}$ 薄膜, 多次沉积可增加薄膜厚度。Tomas[45]发现 $\mathrm{Fe}(\mathrm{CO})_{5}$ 沉积的铁薄膜中 $\mathrm{Fe}$ 的元素含量稳定在 $90 \%$ 以上, 具 有较高的纯净度。但羰基铁的空气和热敏性均较差, 且制 备及储存不便性限制了其应用。张[46]等人改进了二茂铁 的使用方法, 通过将二茂铁在 $60^{\circ} \mathrm{C} \sim 80^{\circ} \mathrm{C}$ 升华, 降低喂养 速率, 在硅基底上原位形成了纳米催化剂颗粒, 将碳纳米 管的管径从 $41 \mathrm{~nm}$ 降低到 $31 \mathrm{~nm}$ 。同时他还指出铁原子的气 相浓度可以通过二茂铁的喂养速率来控制。随后, Yang[47] 等人进一步研究表明改变二茂铁的沉积量可以使纳米铁 颗粒获得不同分布的情况, 从而实现碳纳米管阵列形貌和 结构的控制, 如图2。Saito[48]通过分析二茂铁的热分解模 型预测: 铁纳米颗粒的粒径与分解温度以及距离管中心径 相距离呈正相关关系,如图3。

MOCVD制备催化剂的一大优势在于成本低, 但缺点 也很明显, 金属有机化合物的气化和产物的粒径控制仍然 是一大难题。而且由于金属有机化合物中含有金属-碳键, 最终产物无法避免地含有一定量金属碳化物, 有可能影响 其催化性能。目前的解决方法是在金属有机化合物热解的 同时引入氢气、氧气或水蒸气, 来减弱含碳基团的分解。 另外, 原位制备催化剂纳米颗粒往往有较高的工艺温度 $\left(500-1200^{\circ} \mathrm{C}\right)$, 无可避地导致纳米催化剂颗粒因烧结而 变大。

\subsection{2. 旋涂法（Spin Coating）}

涂转法 [49]是在金属盐溶液或者酒精溶液中加入粘合 剂后, 用基底在旋涂机上按照一定转速和时间旋涂, 经过 烘烤后获得一定厚度的金属氧化物薄膜。
李照涁[50]等改进了旋涂法, 通过聚苯乙烯 (PS) 微 球模板获得规则排布的催化剂结构。旋涂转移法主要原理 如图所示, 将单层的PS微球模板涂在石英玻璃片上, 然后 将旋涂有微球的石英玻璃以一定角度放入金属盐溶液中, 单层的PS微球模板会脱离石英玻璃而漂浮于溶液液面上, 再用硅片以一定角度从金属盐溶液中将单层PS微球捞出, 就实现了PS微球模板从石英玻璃到硅片祄底上的转移, 此 时金属盐溶液主要集中在了PS微球的缝隙里, 再在一定温 度下加热干燥, 最后在空气中制烧除掉PS微球即可得到规 则排布的催化剂结构。调节PS微球的直径便可改变催化剂 的分布。

\subsection{3. 溶胶凝胶法(Sol-gel)}

溶胶凝胶法 [51]制备催化剂的一般流程是将所用的原 料分散到溶剂中形成低黏度溶液, 将活性组分的多孔先驱 体用一定量金属盐溶液浸渍后，与凝胶结构促进剂按照一 定比例混合反应后形成凝胶。将湿凝胶滴于洁净基底上利 用匀胶机在基底表面形成均匀的催化剂先驱体薄膜, 在通 过㷽烧, 氢气退火等工艺形成具有催化活性的催化纳米颗 粒。溶胶凝胶法制备的催化剂纳米颗粒纯净无杂质，粒径 及表面分布均匀, 通过调节凝胶工艺中Fe的浓度以及匀胶 工艺还可以改变催化剂的分布及粒径, 降低了工艺成本。

林青等人[52]用 $\mathrm{Fe}\left(\mathrm{NO}_{3}\right)_{3}$ 、正硅酸乙酯、无水乙醇按 16: 25: 32的比例混合制备了铁催化剂先驱体。研究还发 现在相同匀胶工艺和退火处理的条件下, 随着铁浓度的增 加, 硅表面 $\mathrm{Fe}$ 的粒径增加, 且面分布趋于均匀化; 当浓度 达到2.5M时, 颗粒发生团聚, 分布也不在密集。随后研究 表明当转速从 $2500 \mathrm{rpm}$ 增加到 $4500 \mathrm{rpm}$, 催化剂的厚度依次 减小。

欧阳雨 [53]和牛志强[54]等人通过配比不同的金属盐 溶液利用溶胶凝胶法制备出三合金碳纳米管催化剂。

\subsection{4. 其他方法}

除此之外还有共沉淀法 [55], 浸渍法 [56],燃烧法 [57], 溶液法[58]等。

\section{4. 退火处理}

基底表面的金属催化剂由于较薄极易氧化, 氧化态催 化剂流动性较低不易形成纳米状颗粒 [59], 尤其对于PVD 法制备的催化剂薄膜, 往往需经过退火处理。退火处理对 催化剂做还原处理并不表示氧化态的催化剂不具备催化 性, 研究已证明金属氧化态同样具备良好的催化能力 [60]。 退火处理[61]改变金属原子的表面能来改变其迁移率, 促 使薄膜状催化剂裂解成为纳米颗粒便于碳纳米管的成核。 预处理的结果将直接影响到催化剂纳米颗粒的粒径、面密 度及催化活性, 最大程度上决定碳纳米管阵列生长的形貌 和结构。 



图2 二茂铁分解后基底表面形貌，负载量:(a)25,(b)50,(c)100,(d)150,(e)200,(f)300mg; (g)碳纳米管阵列的高度以及(h)碳纳米管内外径与二茂铁负载量 关系; [47]。

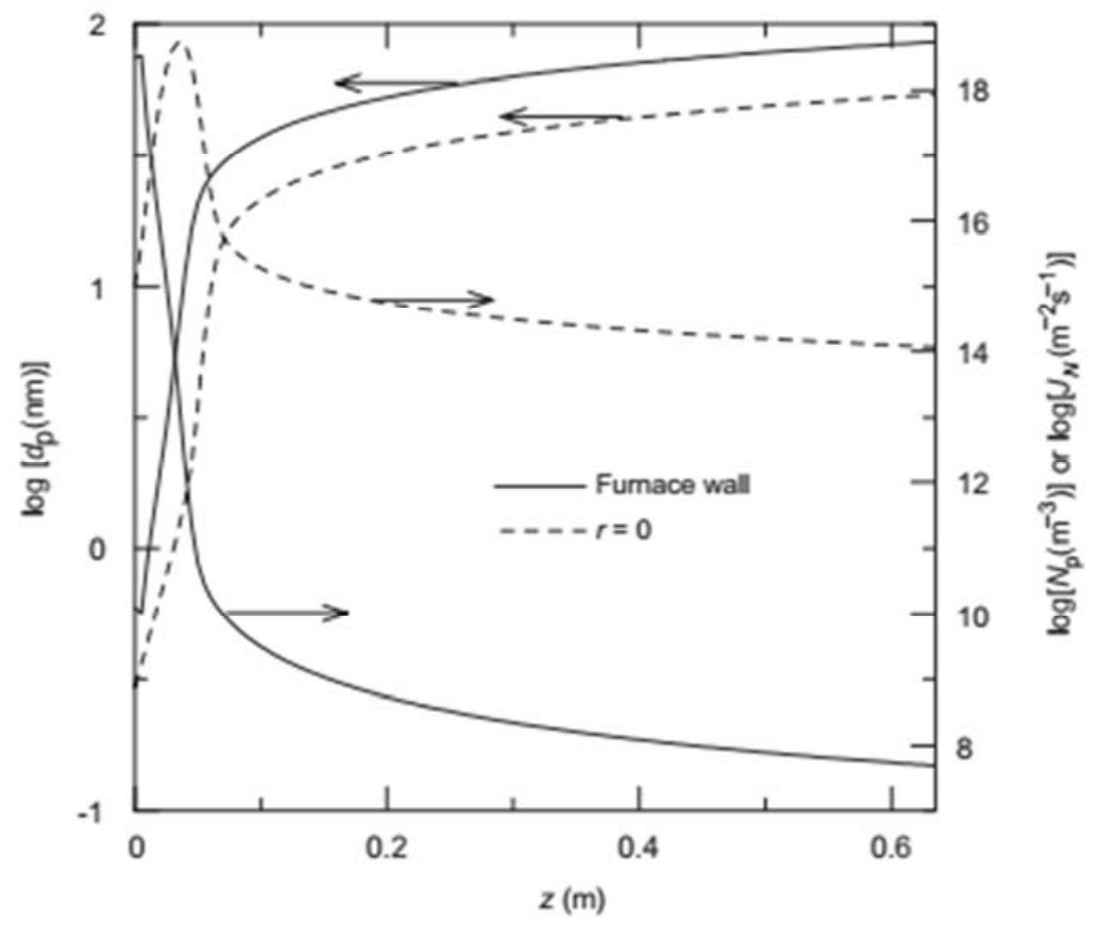




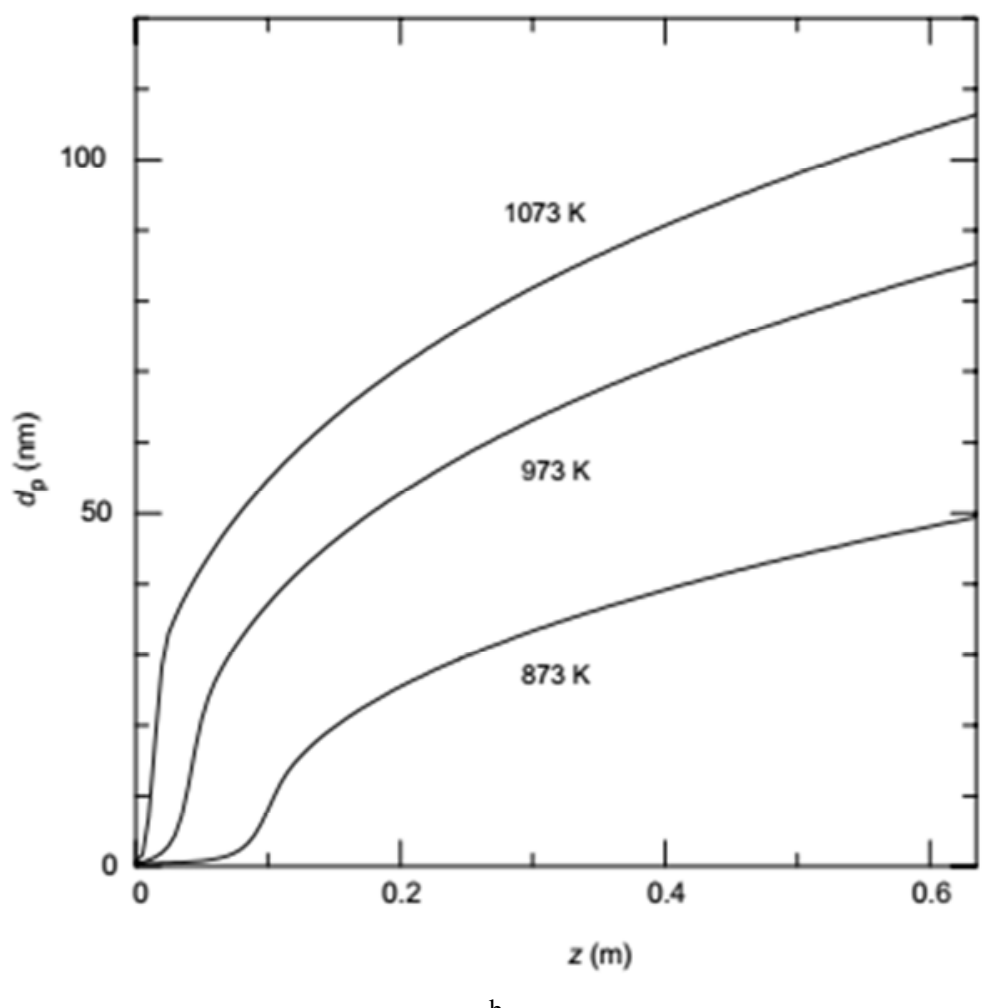

$\mathrm{b}$

图3 (a)颗粒粒径及颗粒密度管沿轴向分布规律：虚线：管径向中心; 实线：管壁； (b)不同温度下管壁轴向铁颗粒粒径分布[48]。
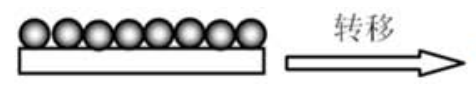
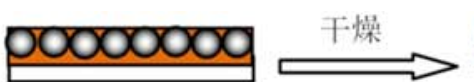

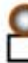

00000009

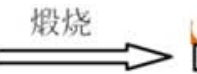

图4 旋涂转移法流程图[50]。

\section{1. 时间}

预处理时间对催化剂的作用主要表现在催化剂尺寸 及分布。Gilbert[62]等人研究氢气处理对氧化铝过渡层上 铁催化剂的“脱浸润”作用时, 发现过长处理时间会导致催 化剂纳米颗粒在表面能驱使下从聚集演变为粗化 (图5), 碳纳米管直径随之增加; 而极短的退火处理时间反而会使 得碳纳米管阵列有较高的表观生长速度。另外, 调整引入 氢气和乙烯的相对时间, 能改变催化剂的还原、粗化以及 碳成核的相对速率: 共同引入碳源与氢气, 碳成核会在催 化剂粗化前开始, 这样生长的碳纳米管阵列管径小, 管空 间间距近, 阵列更长。2014年, Kim[63]在用氢气处理喷 雾法制备的铁薄膜时发现“奥斯特瓦尔德熟化延滞”现象, 如图6。笔者认为随着预处理时间的延长氢气产生的氢活 性集团使 $\mathrm{Al}_{2} \mathrm{O}_{3}$ 过渡层开裂，使催化剂纳米颗粒陷入“陷阱” 中, 影响了扩散和粗化。随后, Schweiger $[64]$ 在 $630^{\circ} \mathrm{C}$ 氢 气处理石英基底上 $0.25 \mathrm{~nm}$ 铁薄膜时发现基底表面的催化 剂颗粒, 没有随着处理时间的延长而一致增大, 而是: 窄 粒径分布 $(1 \mathrm{~min}) \rightarrow$ 宽粒径分布 $(2 \mathrm{~min}) \rightarrow$ 粒径减小 $(5 \mathrm{~min})$ $\rightarrow$ 粒径增大, 分布变宽 (7-10min)。ToF-SIMS分析认为: 2-5min现象是由于基地 $\alpha$-石英与 $\mathrm{Fe}_{2} \mathrm{O} 3$ 原子半径及晶格 常数相近导致的表面颗粒向基地的扩散; 而随着时间延长, 石英 $\alpha$ 相向 $\beta$ 相转变, 使得石英基底中的催化剂颗粒在基底 表面上再出现。相同的现象发生在 $\mathrm{SiO}_{2}[65]$ 和 $\mathrm{Al}_{2} \mathrm{O}_{3}[66]$ 相 中。

\section{2. 气氛}

2006年, Pisana[67]在真空, 氩气, 氢气和氨气气氛 下对 $\mathrm{Fe} / \mathrm{SiO}_{2}$ 退火处理时发现: 真空条件下退火颗粒趋向于 聚集生长成粒径分布集中的大颗粒; 而其他三种气氛条件 下退火, 薄膜均会裂解成为小颗粒。其中氨气具有最佳的 退火效果, 纳米颗粒的粒径分布更为均匀也更小。另外, 增大气氛压力会使催化剂颗粒高度降低, 粒径分布更窄, 适用于单壁碳纳米管的制备。 $\mathrm{H}_{2}$ 和 $\mathrm{NH}_{3}$ 都能保持金属催化 剂的低氧化态以及为催化剂裂解成纳米颗粒提供能量。 June[68] 认为 $\mathrm{NH}_{3}$ 在退火处理中除了上述作用外, 高温下分 解出的 $\mathrm{N}$ 原子还能和金属催化剂形成合金, 促进催化剂中 石墨层的形成和析出。

气氛对催化剂的影响除了颗粒尺寸和形貌, 还可能通 过影响催化剂的结构进而改变 CNT 的性质。例如, Harutyunyan[69]巧妙地设计的 $\mathrm{Ar} / \mathrm{He} / \mathrm{H}_{2} / \mathrm{H}_{2} \mathrm{O}$ 预处理气体 有效提高了金属性SWCNTs的比例。2014年, Bayer[70] 用 $\mathrm{Co} / \mathrm{SiO} 2$ 的催化生长了手型和管径分布都比较集中的 SWCNTs, 变因的唯一解释是氨气预处理对催化剂价态及 颗粒粒径分布的影响。 

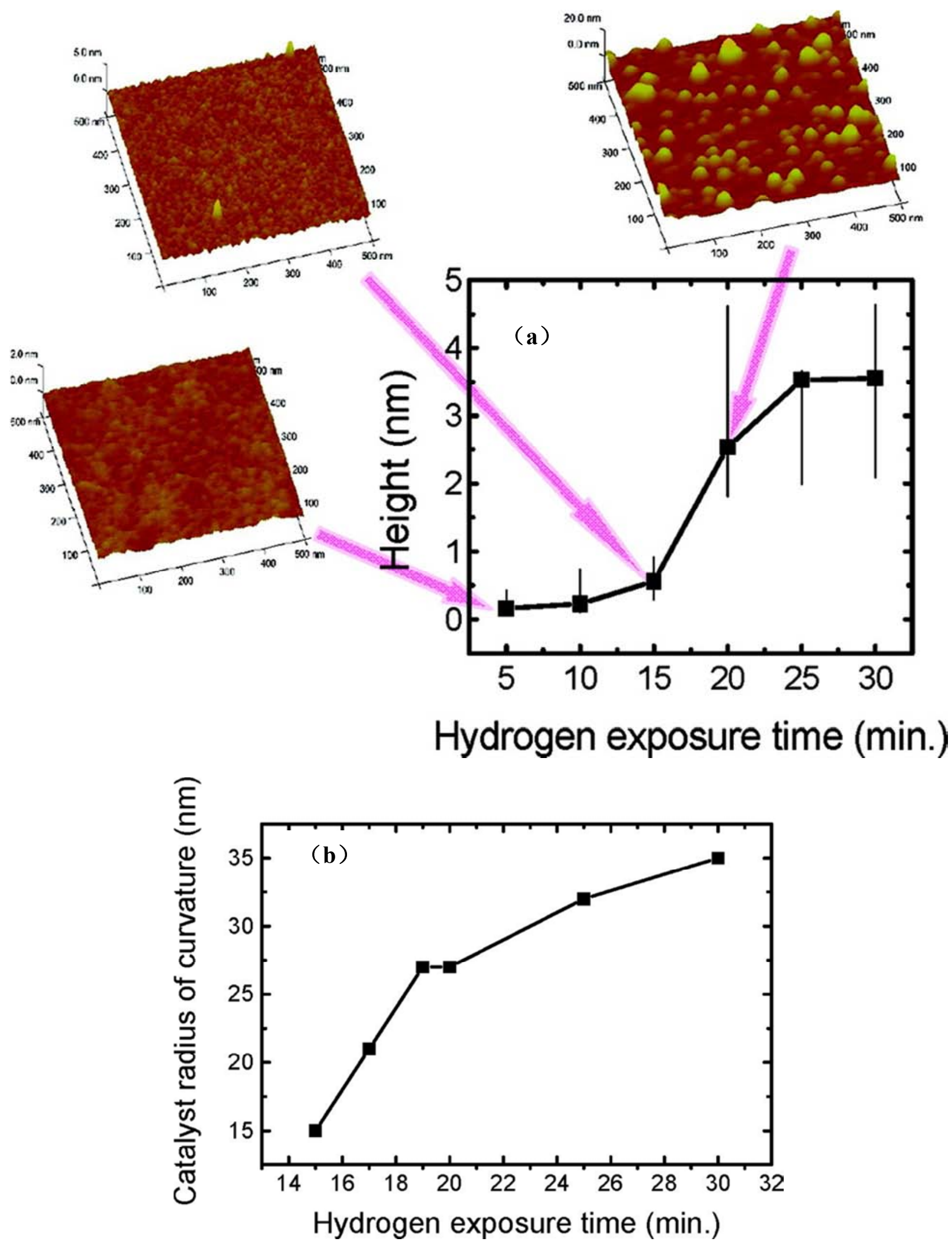

图5 (a)铁原子力显微镜图及团簇高度随着 $770^{\circ} \mathrm{C}$ 氢气气氛下处理时间的变化规律; (b)氢气处理时间与催化剂颗粒粒径关系 [62]。 

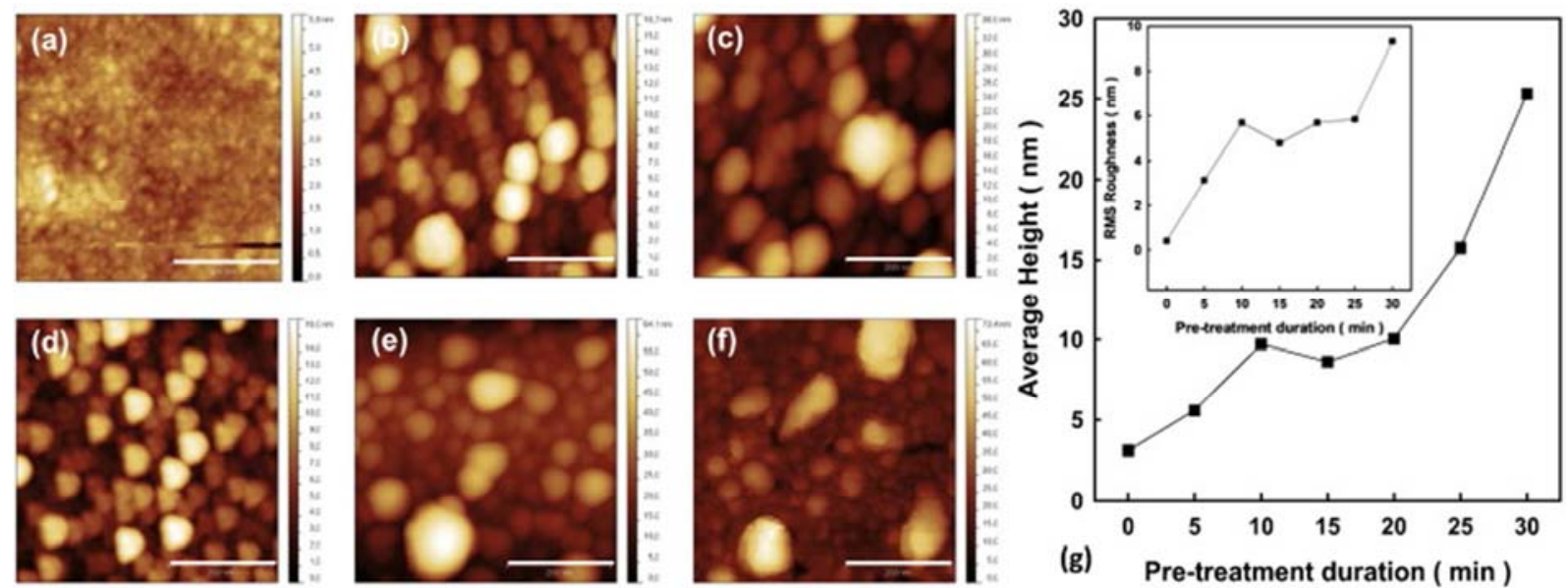

图6 Fe/ $\mathrm{Al}_{2} \mathrm{O}_{3}$ 氢气处理过程中的“奥斯特瓦尔德熟化延滞”现象.(a)-(f)基底表面 $\mathrm{Fe}$ 纳米颗粒随处理时间原子力显微镜图; (g)处理时间与铁纳米团簇尺寸 关系图[63]。

\section{3. 温度}

2003年，Carey[71]在低温 $\left(300-500^{\circ} \mathrm{C}\right)$ 氮气气氛下 处理 $\mathrm{Ni} / \mathrm{TiO}_{2}$ 时发现随着处理温度的升高催化剂小颗粒聚 集成大粒径颗粒薄膜，他通过比较 $\mathrm{Ni}$ 和 $\mathrm{TiO}_{2}$ 的晶格匹配性 及热膨胀系数发现温度升高不会导致颗粒性状的变化, 这 说明高温退火导致小团簇减少的根本原因是表面原子的 迁移。Bouanis[72]氢气预处理 $0.05 \mathrm{~nm} \mathrm{Ru}$ 薄膜时也发现, 当预处理温度从 $850^{\circ} \mathrm{C}$ 升高到 $950^{\circ} \mathrm{C}$, 纳米颗粒从 $1.3 \mathrm{~nm} \pm 0.3 \mathrm{~nm}(78 \%)$ 增加到平均粒径 $4.5 \mathrm{~nm} \pm 0.4 \mathrm{~nm}$, 同时颗 粒的面密度下降。表明预处理温度的升高, 提高了催化剂 原子的扩散能力。另外, 氢气预处理前的快速升温, 可以 有效防止 CNT生长前催化剂颗粒粒径的增大而延长活性 时间[73]。

\section{5. 过渡层}

在沉积催化剂前, 往往需要提前沉积一层缓冲层。缓 冲层的引入抑制了催化剂颗粒的聚集和向基底扩散,进而 降低了催化剂的粒径并提高了催化剂颗粒在基底表面的 堆积密度。 $\mathrm{Al}_{2} \mathrm{O}_{3} 、 \mathrm{Al}_{2} \mathrm{Ox} 、 \mathrm{SiO}_{2} 、 \mathrm{MgO} 、 \mathrm{TiN}$ 是几种常用 的缓冲层材料。金属催化剂纳米颗粒在过渡层表面的浸润 与其表面能有关 [74], 根据Young方程, $\gamma$ 为对应的界面能, $\mathrm{B}$ 沉积在基底 $\mathrm{A}$ 上, 当浸润角大于 $\pi / 2$ 时, $\mathrm{B}$ 在 $\mathrm{A}$ 基底上以岛 状形式生长沉积（Volmer-Weber生长）。研究发现[75], 二氧化硅作为缓冲层时碳纳米管的管径在 $6 \sim 15 \mathrm{~nm}$,堆积 密度通常在 $1 \times 10^{10} \sim 8 \times 10^{10} \mathrm{~cm}^{-2}$; 相比二氧化硅, 氧化铝缓 冲层更趋于形成致密分布的小粒径催化剂颗粒,阵列的堆 积密度也因此增至 $10^{11} \sim 10^{12} \mathrm{~cm}^{-2}$ 的量级。

Carey[71]在氧化物基底上蒸镀Ni纳米颗粒时发现, 金 属薄膜在氧化物基底表面的形核模式依赖于其与基底的 界面反应, 氧化物形成能更高的金属对界面氧更强的吸引 作用, 而界面的相互作用也越强[76]。例如, Fe[77]氧化 物的热形成能 $(-\Delta \mathrm{H})$ 低于 $\mathrm{SiO}_{2} \quad(133.1 \sim 164.5 \mathrm{~kJ} / \mathrm{g}$ atom $>286.7 \mathrm{~kJ} / \mathrm{g}$ atom), 弱相互作用使 $\mathrm{Fe}$ 按照 Volmer-Weber模型生长。即在沉积薄膜较薄时, Fe颗粒呈 现岛状, 随着厚度的增加, 薄膜变得连续, 如图7。Mattevi 等[78]根据原位XPS图谱发现, 金属 $\mathrm{Fe}$ 在 $\mathrm{Al}_{2} \mathrm{O}_{3}$ 缓冲层上的 界面反应要强于 $\mathrm{SiO}_{2}$, 强烈的界面反应给予了金属 $\mathrm{Fe}$ 在表 面移动更大的限制作用, 造就 $\mathrm{Al}_{2} \mathrm{O}_{3}$ 表面上催化剂颗粒粒 径更加集中的分布以及碳纳米管阵列更高的成核密度。 Chen[79]也通过原位XPS及非原位XPS观察到, $\mathrm{SiO}_{2}$ 界面 与金属Fe有较弱的作用键, 导致了低的碳纳米管密度。

另外, 缓冲层的厚度、结构以及制备工艺也是影响催 化剂及碳纳米管阵列生长的重要因素。Zhang[80]等人研究 证明较厚的 $\mathrm{A} 12 \mathrm{O} 3$ (大于 $20 \mathrm{~nm}$ ) 能引导制备较长的碳纳米 管阵列, 而当厚度达到 $30 \mathrm{~nm}-35 \mathrm{~nm}$ 时制备出的CNTAs 具有 较好的纺织性, 而此时催化剂颗粒面密度以及颗粒间距分 别达到最大和最小。可能的原因是氧化铝过渡层对铁原子 或颗粒向基底的扩散效应及表面扩散长大效应的抑制作 用的平衡。 $\mathrm{Han}[81]$ 等通过处理 $\mathrm{Fe} / \mathrm{Al}_{2} \mathrm{O}_{3} / \mathrm{SiO}_{2}$ 中第二缓冲层 $\mathrm{SiO}_{2}$ 的厚度和微观形貌, 获得平均管径 $5 \sim 7 \mathrm{~nm}$ 的单壁碳纳 米管阵列。Chen等[82]设计了三层结构的催化剂/缓冲层结 构 $\mathrm{Al}(0.5-2 \mathrm{~nm}) / \mathrm{Fe}(1.0-2.5 \mathrm{~nm}) / \mathrm{Al}_{2} \mathrm{O}_{3}(5 \mathrm{~nm})$, 生长出长度达到 毫米级的致密垂直单壁碳纳米管阵列, 复合结构中 $\mathrm{Al}_{2} \mathrm{O}_{3}$ 能够抑制催化剂扩散的, 顶层 $\mathrm{Al}$ 使得 $\mathrm{Fe}$ 颗粒在退火处理和 碳管生长过程中保持较小尺寸和高密度。Betty[83]发现不 同制备工艺获得的表面粗䊁度有差异的二氧化硅缓冲层 可以对碳纳米管阵列的控制制备 (粗䊁度: 热氧化 $<\mathrm{ALD}<\mathrm{MPE}-\mathrm{CVD}$ ), 随着粗糙度的增大, 碳纳米管阵列的 高度增加。除了上述影响因素, Tsuji[84]还发现磁控溅射 制备的 $\mathrm{MgO}$ 缓冲层在 $650^{\circ} \mathrm{C}$ 下进行了退火处理, 使得 $\mathrm{Fe}$ 催 化剂的活性时间从 $2 \mathrm{~min}$ 延长到 $10 \mathrm{~min}$ 以上, 并且制备出阵 列长度由 $0.1 \mathrm{~mm}$ 增长到 $1.5 \mathrm{~mm}$ 的单壁碳纳米管含量为 $95 \%$ 的阵列。XPS光谱分析认为, 这种提升得益于预处理的缓 冲层对催化剂纳米颗粒的表面扩散的抑制以及对金属价 态的稳定。毫无疑问, 这对选择缓冲层材料来提高阵列生 长效率有重要指导意义。 


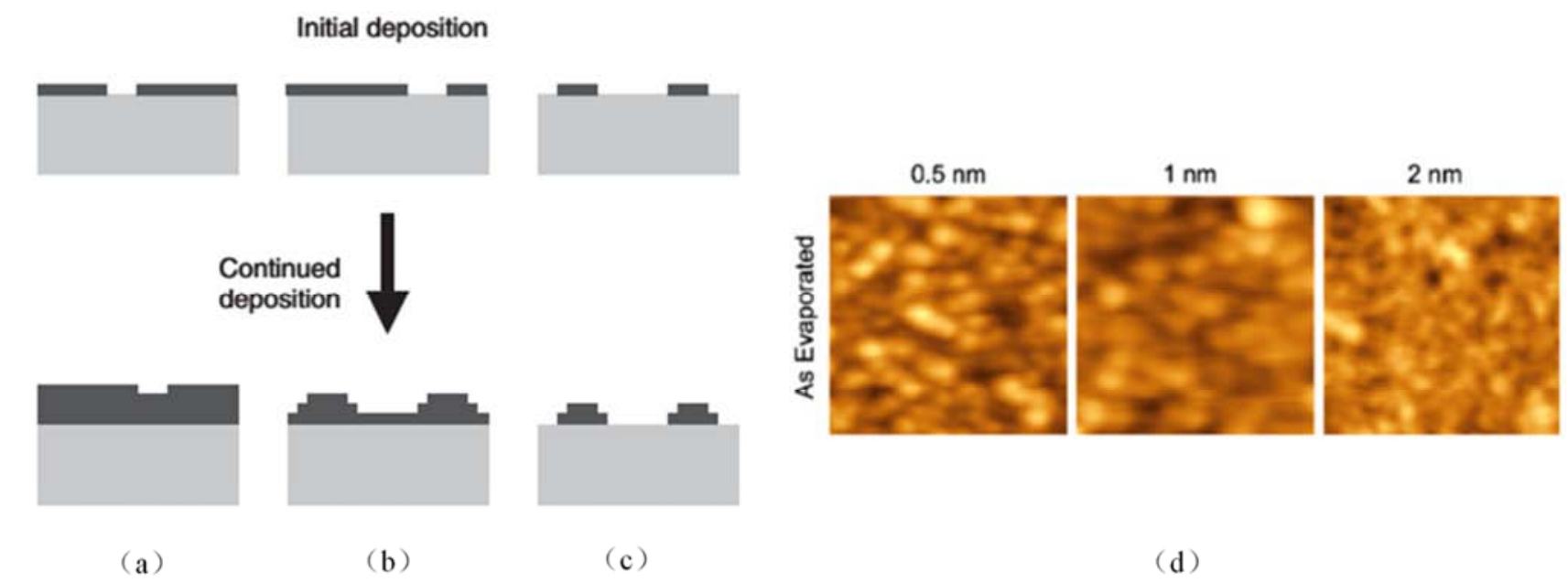

图7 金属-氧化物的生长模型显示其最初的浸润行为及随后薄膜的沉积[71] (a)强界面作用:2D Frank-van der Merwe layer-by-layer模型; (b):StranskiKrastanow模型; (c):弱界面作用:2D Volmer-Weber模型; (d)不同厚度的铁薄膜AFM图[67]。

\section{6. 结论}

目前, 碳纳米管的宏观制备已经达到了千吨级的年产 能。尽管如此，对于单根碳纳米管的控制和稳定质量相关 的实践经验仍然缺乏。大批量可控制备形貌结构优异的垂 直碳纳米管阵列仍旧是未来研究的重点和难点, 尤其碳纳 米管的手性、缺陷、全同长度与壁数等方面的控制。深入 理解碳纳米管的催化控制机理是实现这些目标的关键。

本文已就由催化剂的制备方法、退火处理工艺以及催 化剂和缓冲层的选取导致的催化剂纳米颗粒的形貌分布、 价态等变化对碳纳米管阵列的影响进行了说明。总结前人 的研究发现, PVD工艺制备的催化剂层相较于化学方法在 形貌、结构及随后的处理的可控性上具有明显的优势, 而 化学法对催化剂化学成分的控制可控性较强。退火处理时 间、温度、气氛以及缓冲层对催化剂的作用除了对化合价 产生影响外, 主要体现在催化剂在表面层的扩散以及向基 底层的扩散动力学上。

\section{参考文献}

[1] M.麦亚潘.碳纳米管-科学与应用 $[\mathrm{M}]$, 北京, 科学出版社, 2007 。

[2] A E Aliev, M E Kozlov, A A Kuznetsov, et al. Giant-stroke, super elastic carbon nanotube aerogel muscles[J], Science, $2009,323,1575-1578$.

[3] P Simon, Y Gogotsi, Materials for electrochemical capacitors[J], Nature. Materials. 2008, 7(11): 7845-7854.

[4] Zu-Po Yang, Lijie Ci, James A. Bur, S-Y Lin, et al., Experimental Observation of an Extremely Dark Material Made By a Low-Density Nanotube Array[J], Nano Letter, 2008, 8(2): 446-451.

[5] Yang Liu, Yichun Zhang, Xu Wang, et al., Excel-lent Microwave Absorbing Property of Multiwalled Carbon Nanotubes with Skin-Core Heterostructure Formed by Outer
Dominated Fluorination[J], The Journal of Physics Chemical C, 2018, 122(11):6357-6367.

[6] Lin Qiu, Kimberly Scheider, RA Suhaib, Optimized Growth of Muti-walled Carbon Nanotube Arrays for Thermal interface Applications[J], Journal of Engineering Thermophysics, 2017, 38(6): 1323-1327.

[7] Zhang M, Fang S, Zakhidov A A, et al. Strong, transparent, multifunctional, carbon nanotube sheets[J]. Science, 2005, 309(5738): 1215-1219.

[8] Feng C, Liu K, Wu J S, et al. Flexible, stretchable, transparent conducting films made from superaligned carbon nanotubes. Advanced Functional Materials, 2010, 10(6): 885-891.

[9] Kohei Mizuno a, Juntaro Ishii b, Hideo Kishida, et al. A black body absorber from vertically aligned single-walled carbon[J]. PNAS, 2009, 106(15):6044-6047.

[10] Zu-Po Yang, Mei-Li Hsieh, James A. Bur, et al. Experimental observation of extremely weak optical scattering from an interlocking carbon nanotube array[J], Applied Optics, 2011, 50(13): 1850-1855.

[11] Zhe Yin, Huimin Wang, Muqiang Jian, et al., Extremely Black Vertically Aligned Carbon Nanotube Arrays for Solar Steam Generation[J], APPLIEDMATERIALS \& INTERFACES, 2017, 9(34):28696-28603.

[12] Lei Zhang, Bin Zhao, Chuan Jiang, et al., Preparation and Transport Performances of High-Density, Aligned Carbon Nanotube Membranes[J], Nanoscale Research Letters, 2015, 10:266.

[13] S. S. Fan, M. G. Chapline, N. R. Franklin, et al. Self-oriented regular arrays of carbon nanotubes and their field emission properties[J], Science, 1999, 283 (5401):512-519.

[14] K Hata, D N Futaba, K Mizuno, S Iijima, et al., Water-assisted highly efficient synthesis of impurity-free single-walled carbon nanotubes[J], Science, 2004, 306:1362-1364.

[15] C Mattevi, C T Wirth, S Hofmann, et al. In-situ X-ray photoelectron spectroscopy study of catalyst-support interactions and growth of carbon nanotube forests[J], The Journal of Physics Chemical C, 2008, 112, 12207-12213. 
[16] B Kitiyanan, W E Alvarez, J H Harwell, et al. Controlled production of single-wall carbon nanotubes by catalytic decomposition of $\mathrm{CO}$ on bimetallic Co-Mo catalysts[J], Chemical. Physics Letter, 2000, 317, 497-503.

[17] Qi Li, Anderson D. Smith, Mazharul Haque, et al. Graphite paper/carbon nanotube composite:A potential supercapacitor electrode for powering microsystem technology[A]. Journal of physics: Conference Series. 2017, 922(1):1-6.

[18] Linquan Ping, Peng-Xiang Hou, Han Wang, et al,. Clean, fast and scalable transfer of ultrathin/patterned vertically-aligned carbon nanotube arrays[J], Carbon, 133:275-282.

[19] Shota Miura, Yu Yoshihara, Mayu Asaka, Kei Hasegawa, et al., Millimeter-tall carbon nanotube arrays grown on aluminum Substrates[J], Carbon, 2018, 130:834-842.

[20] Moisala A, Nasibulin AG, Kauppinen EI. The role of metal nanoparticles in the catalytic production of single-walled carbon nanotubes-a review[J], Journal of Physics: Condens Matter 2003, 15: S3011-3035.

[21] Dupuis AC. The catalyst in the CCVD of carbon nanotubes-a review[J]. Progress in Material Science, 2005, 50(8):929-961.

[22] Irle S, Ohta Y, Okamoto Y, et al. Milestones in molecular dynamics simulations of single- walled carbon nanotube formation: a brief critical review[J]. Nano Res, 2009, 2(10):755-767.

[23] Hong G, Chen YB, Li P, Zhang J. Controlling the growth of single-walled carbon nanotubes on surfaces using metal and non-metal catalysts[J]. Carbon, 2012, 50(6):2067-2082.

[24] Tessonnier JP, Su DS. Recent progress on the growth mechanism of carbon nanotubes: a review $[\mathrm{J}]$. Chemsuschem, 2011, 4(7):824-47.

[25] Xu J, Saeys M. First principles study of the stability and the formation kinetics of subsurface and bulk carbon on a $\mathrm{Ni}$ catalyst. J Phys Chem C 2008; 112 (26) :9679-85.

[26] Wei-Qiao Deng, Xin Xu, and William A. Goddard, A Two-Stage Mechanism of Bimetallic Catalyzed Growth of Single-Walled Carbon Nanotubes[J]. Nano Letter, 2004, 4(12):2331-2335

[27] Yuki Matsuoka and Masamichi Yoshimura, Effect of catalytic metals of various elements on synthesis of graphite-capped, vertically aligned carbon nanotube arrays[J], Japanese Journal of Applied Physics, 2014, 53(4):045501.

[28] Yang W, Feng Y, Chu W, Catalytic chemical vapor deposition of methane to carbon nanotubes: copper promoted effect of $\mathrm{Ni} / \mathrm{MgO}$ catalysts[J]. Journal of Nanotechnology, 2014, 1-5.

[29] A J Hart, A H Slocum, Rapid growth and flow-mediated nucleation of millimeter-scale aligned carbon nanotube structures from a thin-film catalyst[J], Journal of Physics Chemical B, 2006, 110 (110):8250-8257.

[30] Takeo Yamada, Tatsunri Namai, Kenji Hata, et al. Size-selective growth of double-walled carbon nanotube forests from engineered iron catalysts[J], Nature nanotechnology, 2006, 1(2): 131-136.

[31] Seung-Woong Lee, Jaeseok Han and Hyunsik Im, Facile Method to Control the Diameter and Density of Carbon
Nanotubes by Using a Catalyst-embedded Supporting Layer[J], Journal of the Korean Physical Society, 2015, 66(11): $1715-1719$.

[32] Mandar Kini, Stefan E Schulz, Effects of catalyst configurations and process conditions on the formation of catalyst nanoparticles and growth of single-walled carbon nanotubes[J], Microelectronic Engineering, 2016, 167:95-104.

[33] N. Bouts, M. Gaillard, L. Donero, et al. Growth control of carbon nanotubes using nanocomposite nickel/carbon thin films[J], The Solid Films, 2017, 630:38-47.

[34] Toshiyuki Ohashi, Toshiyuki Shima, Synthesis of vertically aligned single-walled carbon nanotubes with metallic chirality through facet control of catalysts[J], Carbon, 2015, 87: 453-461.

[35] M. Knez, K. Nielsch, and L. Niinisto, Synthesis and surface engineering of complex nanostructures by atomic layer deposition[J], Advanced Materials. 2007, 19(21):3425-3438.

[36] DRG. Mitchell, DJ Attard, JR Bartlett, TEM and ellipsometry studies of nanolaminate oxide films prepared using atomic layer deposition[J], Applied Surface Science, 2005, 243(1):265-277.

[37] Bingan Chen, Can Zhang, Santiago Esconjauregui, et al. Carbon nanotube forests growth using catalysts from atomic layer deposition[J], Journal of Applied Physics, 2014, 115(14):878.

[38] YANG Chao, LI Ying, YAN Lu, Influence of Catalyst Film Thickness Deposited by Atomic Layer Deposition on Growth of Aligned Carbon Nanotubes[J], Journal of Inorganic Materials, 2016, 36(17): 681- 686.

[39] Lei Tang, Qichong Zhang, Chaowei Li, et al. Atomic layer deposition of $\mathrm{Al}_{2} \mathrm{O}_{3}$ catalysts for narrow diameter distributed single-walled carbon nanotube arrays growth[J], Carbon, 2016 11547.

[40] 田民波, 李正操, 薄膜技术与薄膜材料 [M],清华大学出版 社, 2011。

[41] Naoki Yoshikawa, Takuma Asari, Naoki Kishi, et al. An efficient fabrication of vertically aligned carbon nanotubes on flexible aluminum foils by catalyst supported chemical vapor deposition[J], Nanotechnology, 2008, 19(24):245607.

[42] Kai Zhang, Taotao Li, Lin Ling, et al., Facile synthesis of high density carbon nanotube array by a deposition -growth -densification process[J], Carbon, 2017, 114:435-440.

[43] Douglas K Russell, Gas-Phase Pyrolysis Mchanisms in Organometallic CVD[J], Chemical Vapor Deposition, 1996, 2(6): 223-233.

[44] On Mero, Efrat Shawat, Gilbert D, et al. Air-stable nanogranular $\mathrm{Fe}$ thin films formed by Chemical Vapor Deposition of triiron dodecacarbonyl as catalysts for carbon nanotube growth[J], Thin Sold Films. 2014, 550:76-84.

[45] Ioannis G Aviziotis, Thomas Duguet, Combined Macro / Nanoscale Investigation of the Chemical Vapor Deposition of $\mathrm{Fe}$ from $\mathrm{Fe}(\mathrm{CO})_{5}[\mathrm{~J}]$, Advanced Materials Interfaces, 2017, 4(18): 1601185. 
[46] Qiang Zhang, Jia-Qi Huang, Meng-Qiang Zhao, et al. Modulating the diameter of carbon nanotubes in array form via floating catalyst chemical vapor deposition[J], Applied Physics A, 2009, 94(4) : 853-860.

[47] Xiaoshuang Yang, Lixiang Yuan, Vanessa K, et al. Facile Preparation of Free-Standing Carbon Nanotube Arrays Produced Using Two-Step Floating-Ferrocene Chemical VaporDeposition[J], AppliedMaterials \& Interfaces, 2012, 4(3) : 1417-1422.

[48] Kazunori Kuwana, Kozo Saito, Modeling CVD synthesis of carbon nanotubes:Nanoparticle formation from ferrocene[J], Carbon, 2005, 43(10):2088-2095.

[49] HJ Kim, Eugene Oh, Jaegeun Lee, KH Lee, Synthesis of single-walled carbon nanotubes using hemoglobin-based iron catalyst[J], Carbon, 2011, 50(2): 722-726.

[50] 李照涁, 基于光伏碳纳米管阵列的制备及其陷光性能初步 研究 $[\mathrm{M}]$,燕山大学, 2016.5。

[51] Dawei Li, Lujun Pan, Juanjuan Qian, et al. Highly efficient synthesis of carbon nanocoils by catalyst particles prepared by a sol-gel method[J], Carbon, 2010, 48(1):170-175.

[52] 林青, 贺琪, 化学气相沉积法制备碳纳米管有序阵列 [J], 青岛大学学报（工程技术版），2008，3：23-29。

[53] 欧阳雨,刘启金金, Mo-Fe催化剂中Mo含量对SWCNTs的制 备影响 [J],临沂师范学院学报,2007,29(6):31-35。

[54] 牛志强, 方炎, 催化剂组分对制备单壁碳纳米管的影响 [J], 物理学报, 2007, 56(3):1796-1801。

[55] Chunhua Li, Kefu Yao, Ji Liang, Study on the features of multiwalled carbon nanotube upported nickel aluminum mixed oxides[J], Applied Catalysis A:General, 2004, 261(2):221-224.

[56] NIE Jing-Qi, ZHANG Qiang, ZHAO Meng- Qiang, et al. Synthesis of high quality singlewall carbon nanotubes on natural sepiolite and their use for phenol absorption[J]. Carbon. 2011, 49: 1568-1580.

[57] TANG S, ZHONG Z, XIONG Z, et al. Controlled growth of single-walled carbon nanotubes by catalytic decomposition of $\mathrm{CH}_{4}$ over $\mathrm{Mo} / \mathrm{Co} / \mathrm{MgO}$ catalysts[J]. Chemical Phrsics Letters, 2001, 350: 19-26.

[58] Kiyofumi Yamagiwa, Yusuke Ayato, Jun Kuwano , Liquid-phase synthesis of highly aligned carbon nanotubes on preheated stainless steel substrates[J], Carbon, 2016, 98:225-231.

[59] Gilbert D. Nessim, A. John Hart, Jin S. Kim, et al. Tuning of Vertically-Aligned Carbon Nanotube Diameter and Areal Density through Catalyst Pre-Treatment[J], Nano Letters, 2008, 8 (11): 3587-3593.

[60] Eti Teblum, Yossi Gofer, Cary L Pint, et al. Role of Catalyst Oxidation State in the Growth of Vertically Aligned Carbon Nanotubes[J], The Journal of Physical Chemistry C, 2012, 116(46): 24522-24528.

[61] Sharma R, Chee SW, Herzing A, Miranda R, Rez P. Evaluation of the role of $\mathrm{Au}$ in improving catalytic activity of
Ni nanoparticles for the formation of one-dimensional carbon nanostructures[J]. Nano Letter 2011;11(6):2464-671.

[62] Gilbert D. Nessim, A. John Hart, Jin S. Kim, et al, Tuning of Vertically-Aligned CarbonNanotube Diameter and Areal Density[J], Nano Letters, 2008, 8(11), 3587-3593.

[63] KH Kim, Aurélien Gohier, Jean-Eric Bouree, et al. The role of catalytic nanoparticle pretreatment on the growth of vertically aligned carbon nanotubes by hot-filament chemical vapor deposition[J], Thin Solid Flims, 2015, 575(23), 84-91.

[64] Manuel Schweiger a, Maximilian Schaudig a, Florentina Gannott, et al., Controlling the diameter of aligned single-walled carbon nanotubes on quartz via catalyst reduction time[J], Carbon, 2015, 95:452-459.

[65] S. Sakurai, H. Nishino, D. N. Futaba, S. Yasuda, T. Yamada, A. Maigne, et al., Role of subsurface diffusion and Ostwald ripening in catalyst formation for singlewalled carbon nanotube forest growth, J. Am. Chem. Soc. 2012, 134 (4) :2148-2153.

[66] Y. Hu, L. Kang, Q. Zhao, H. Zhong, S. Zhang, L. Yang, et al., Growth of highdensity horizontally aligned SWNT arrays using Trojan catalysts, Nature Communication, 2015, 6: 6099.

[67] S. Pisana, M. Cantoro, A. Parvez, et al., The role of precursor gases on the surface restructuring of catalyst films during carbon nanotube growth[J], Physica E, 2007, 37:1-5.

[68] Minjae J, Kwang Y E, Young-Joon B, et a1. Effect of $\mathrm{NH}_{3}$ environmental gas on the growth of aligned carbon nanotube in catalystically pyrolizing $\mathrm{C}_{2} \mathrm{H}_{2}[\mathrm{~J}]$. Thin Solid Films, 2001, 398: 150-155.

[69] Harutyunyan A R, Chen G, Paronyan T M, et al. Preferential Growth of Single-Walled Carbon Nanotubes with Metallic Conductivity, Science, 2009, 326, 116-120.

[70] Martin Fouquet, Bernhard C Bayer, Santiago Esconjauregui, Effect of Catalyst Pretreatment on Chirality-Selective Growth of Single-Walled Carbon Nanotubes[J], The Journal of Physical Chemistry C, 2014, 118(11):5773-5781.

[71] J D Carey, L L Ong and S R P Silva, Formation of low-temperature self-organized nanoscale nickel metal islands[J], Nanotechnology, 2003, 14(11): 1223- 1227.

[72] F Z Bouanis, I Florea, M Bouanis, D Muller, et al., Diameter controlled growth of SWCNTs using Ru as catalyst precursors coupled with atomic hydrogen treatment[J], Chemical Engineering Journal, 2017, 332: 92-101.

[73] Zhaoli Gao, Xinfeng Zhang, Kai Zhang, and Matthew M F Yuen, Growth of Vertically Aligned Carbon Nanotube Arrays on Al Substrates through Controlled Diffusion of Catalyst[J], The Journal of Physical Chemistry C, 2015, 119(27):1-35.

[74] Venables J A. The chemical physics of solid surfaces. In:King DA, Woodruff DP, editors. Growth and properties of ultrathin epitaxial layers, 1997, 8, 1-45.

[75] Futaba D N, Hata K, Namai T, et al., 84\% catalyst activity of water-assisted growth of single walled carbon nanotube forest characteriza-tion by a statistical and macroscopic approach $[\mathrm{J}]$. The Journal of Physics Chemistry B, 2006, 110(15): 80358038 . 
[76] See, for example, Henrich V E and Cox P A 1994 The Surface Science of Metal Oxides (Cambridge: Cambridge University Press) chapter 5.

[77] R. Pretorius, J. M. Harris, M.-A. Nicolet, Solid-State Electron. 21(1978) 667.

[78] Mattevi C, Wirth C T, Hofmann S, et al. In-situ X-ray photoelectron spectroscopy study of catalyst-support interactions and growth of carbon nanotube forests[J]. The Journalof Physical Chemistry C, 2008, 112 (32) : 12207-12213.

[79] Bingan Chen, Can Zhang, Santiago Esconjauregui, et al., Carbon nanotube forests growthusing catalysts from atomic layer deposition[J], Journal of Applied Physics, 2014, 115(14):144303.

[80] Yani Zhang, Gengzhi Sun, Zhaoyao Zhan, and Lianxi Zheng, Influence of $\mathrm{Al} 2 \mathrm{O} 3$ buffer layer on catalyst morphology and spinnability of carbon nanotube arrays[J], J. Mater. Sci., 2017, 52:6196-6204.
[81] Han Z J, Ostrikov K. Uniform, dense arrays of vertically aligned, large-diameter single-walled carbon nanotubes[J]. Journal of the American Chemical Society, 2012, 134 (13): 6018-6024.

[82] Guohai Chen a, Shunsuke Sakurai, Motoo Yumura, et al., Highly pure, millimeter-tall, sub-2-nanometer diameter single-walled carbon nanotube forests[J], Carbon, 2016, 107 : 433-439.

[83] Betty T. Quinton, Kevin D. Leedy, Jacob W. Lawson, et al., Influence of oxide buffer layers on the growth of carbon nanotube arrays on carbon substrates[J], Carbon, 2015, 87:175-185.

[84] Takashi Tsuji, Kenji Hata, Don N. Futaba and Shunsuke Sakurai, Unexpected Efficient Synthesis of Millimeter-Scale Single-Wall Carbon Nanotube Forests Using a Sputtered MgO Catalyst Underlayer Enabled by a Simple Treatment Process[J], Journal of The American Chemical Society, 2016. 